\title{
Diabetes classification using a redundancy reduction preprocessor
}

\author{
Áurea Celeste Ribeiro*, Allan Kardec Barros, Ewaldo Santana, José Carlos Príncipe
}

\begin{abstract}
Introduction Diabetes patients can benefit significantly from early diagnosis. Thus, accurate automated screening is becoming increasingly important due to the wide spread of that disease. Previous studies in automated screening have found a maximum accuracy of $92.6 \%$. Methods: This work proposes a classification methodology based on efficient coding of the input data, which is carried out by decreasing input data redundancy using well-known ICA algorithms, such as FastICA, JADE and INFOMAX. The classifier used in the task to discriminate diabetics from non-diaibetics is the one class support vector machine. Classification tests were performed using noninvasive and invasive indicators. Results: The results suggest that redundancy reduction increases one-class support vector machine performance when discriminating between diabetics and nondiabetics up to an accuracy of $98.47 \%$ while using all indicators. By using only noninvasive indicators, an accuracy of $98.28 \%$ was obtained. Conclusion: The ICA feature extraction improves the performance of the classifier in the data set because it reduces the statistical dependence of the collected data, which increases the ability of the classifier to find accurate class boundaries.
\end{abstract}

Keywords: Diabetes, Clustering, Efficient coding, Independent Component Analysis, Support Vector Machine.

\section{Introduction}

Diabetes is a disease caused by the pancreas failing to produce insulin or when the body cannot effectively process this hormone. Despite an increasing amount of information about the disease, traditional data analysis approaches have become inefficient, and automated methods for efficient extraction of information are essential for diagnosis. It has been suggested that applying machine learning to medical analysis may increase diagnostic accuracy and reduce costs and human resources (Kayaer and Yildirim, 2003).

Many studies have contributed to the improvement of the classification of diabetes by using the Pima Indian database, as is shown in Table 1. For example, Byeon et al. (2008) used genetic algorithms (GAs) and prototyped selection methods with an accuracy rate of $92.60 \%$, which to the best of our knowledge achieves the best performance to date. Patil et al. (2010) used a hybrid prediction model with an accuracy rate of $92.38 \%$. Lee and Wang (2011) used a fuzzy expert system with an accuracy rate of $91.20 \%$, and Polat and Güneş (2007) used principal component analysis (PCA) with an adaptive neuro fuzzy inference system with an accuracy rate of $89.47 \%$. The proposed method adopts the basic idea of Byeon et al., which removes redundancy in a database by means of GAs.

Table 1 also presents other relevant studies, and the general conclusion is that the automatic classification of diabetes, at least for a homogeneous population, can be performed with high accuracy. However, some features are invasive and preclude the general triage of diabetes. Moreover, there is room for improvement, which can result in more confidence in machine learning techniques.

An idea that has been successfully employed is feature extraction prior to classification. This can reduce redundancy in data. Essentially, feature extraction methods can be divided into two approaches. In the first approach, the feature extractor and classifier are trained together, and in the second approach they are trained independently. For example, Hild et al. (2006) proposed an information theoretic methodology in order to select features for any classifier. In this regard, PCA is commonly employed to project the input data to a subspace that preserves maximal power and where the directions associated with smaller eigenvalues can be pruned, which effectively creates an orthogonal space for the features of reduced dimension. However, PCA limits the use of data to second order statistics (Polat and Güneş, 2007). Although other studies used different techniques during the feature extraction phase, such as a GA (Byeon et al., 2008) and linear discriminant analysis (LDA) (Çalişir and Doğantekin, 2011), their results showed reasonable accuracy but did not utilize the full statistical information contained in the features. However, we believe that feature extraction is a key intermediate step in classification because it 
simplifies the design of classifiers. In this paper, we propose an information theoretic approach for feature extraction. The proposed method uses higher order statistical information about the collected data in order to provide a projection space, which, in contrast to PCA, preserves better the information contained in the data cloud. Our method uses independent component analysis (ICA) decomposition. ICA uses higher-order statistical information about input data; thus, it has the potential to improve the separability of classes in the projected space, thus improving the performance of the One-class support vector machines used here (Costa et al., 2011; Lucena et al., 2011; Sample et al., 2005; Smith and Lewicki, 2006).

\section{Methods}

Computer Aided Diagnosis (CAD) techniques may assist in diabetes treatment by preventing complications of the disease. Consequently, they reduce social and economic costs, because diabetes has a decade long asymptomatic phase which, if not diagnosed early, can cause complications (Silva et al., 2014). Therefore, we propose a CAD method with a methodology that can be summarized in the block diagram in Figure 1. The input data (1) are subjected to a feature extraction process (2) to create a new representation of the original data with minimum redundancy. The new representation, according to the extraction based on efficient coding, is the input for the one-class SVM classifier (3) that clusters the two classes. To model efficient coding, three algorithms which are already well tested in the literature were used to find a better way to represent the data: JADE, INFOMAX, and FastICA. In the following sections we will describe the methods used here.

\section{Minimizing statistical dependency with efficient coding}

Sensory processing studies have suggested that neural processes may deal with information by using the efficient coding strategy (Baddeley et al., 1997; Barros and Chichocki, 2002; Deweese et al., 2003; Doi et al., 2003; Hubel and Wiesel, 1962; Simoncelli and Olshausen, 2001). The fundamental assumption here is that data $x$ colected by sensors is an unknown linear instantaneous mixture of unknown independent external stimulus $s$ mixed by an unknown matrix $A$ (i.e., $A$ are the underlying "causes" of the observed characteristics) that were important in the formation of the observed data $x$.

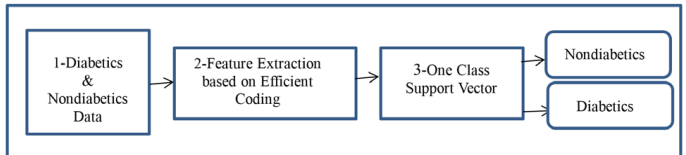

Figure 1. Block diagram of the proposed methodology (Extracting features based on efficient coding + one-class SVM).

Table 1. List of works-related the experiment with all indicators. In these works were used all clinical indicators of the PIMA database (invasive and non-invasive indicators).

\begin{tabular}{ccc}
\hline Method & Accuracy & Reference \\
\hline Proposed method with all indicators & $98.47 \%$ & \\
GA and Prot. Selec. & $92.60 \%$ & Byeon et al. (2008) \\
HPM & $92.38 \%$ & Patil et al. (2010) \\
Fuzzy & $91.20 \%$ & Lee and Wang (2011) \\
LDA-Wavelet SVM & $89.74 \%$ & Çalişir and Doğantekin (2011) \\
IFE-CF & $89.48 \%$ & Reddy and Reddy (2010) \\
PCA-ANFIS & $89.47 \%$ & Polat and Gunes (2007) \\
MAIRS2 & $89.10 \%$ & Chikh et al. (2012) \\
LDA-ANFIS & $84.61 \%$ & Dogantekin et al. (2010) \\
ANN-FNN & $84.24 \%$ & Kahramanli and Allahverdi (2008) \\
GDA-LS-SVM & $82.05 \%$ & Polat et al. (2008) \\
C-HMLP & $81.74 \%$ & Mat Isa and Mamat (2011) \\
Fuzzy & $79.37 \%$ & Lekkas and Mikhailov (2010) \\
ANFIS & $77.65 \%$ & Ghazavi and Liao (2008) \\
Fuzzy & $77.8 \%$ & Luukka (2011a) \\
ANN & $76.62 \%$ & Jeatrakul et al. (2010) \\
OP-ELM & $76.3 \%$ & Miche et al. (2010) \\
IP-LSSVM & $76.1 \%$ & Carvalho and Braga (2009) \\
FSM-FuzzyEM & $75.97 \%$ & Luukka (2011b) \\
SVM & $75.15 \%$ & Li and Liu (2010) \\
\hline
\end{tabular}


The idea behind efficient coding is that the collected data is a combination of causes or basis functions that, in turn, produce the observations. Because the combined data usually becomes more redundant, the goal is to undo this increase in statistical dependence by performing ICA on the collected data. Although the space might be composed of nonorthogonal basis functions belonging to $A$, the projections $s$ are statistically independent. This is where ICA appeals to efficient coding. Here, let $x=\left(x_{1}, x_{2}, x_{3}, \ldots, x_{n}\right)$ be a set of observations taken from the same data. Using $x=A s$ as training input, ICA learns the basis functions in the columns of a matrix $A$ for the features such that the variables comprising vector $s$ are mutually statistically independent (Hyvärinen and Oja, 2000; Comon, 1994).

There are several ways to estimate the matrix, $W$ where $W=A^{-1}$ from the projection phase $s=A^{-1} x$, and each method involves different algorithms. We have used three of the most used algorithms in our tests. These algorithms are reviewed in the following sections.

\section{FastICA algorithm}

The FastICA is a computationally efficient and well-tested algorithm. We assume that vector $x$ is whitened by PCA, which is typical in ICA literature (Hyvärinen et al., 2001). The weight vector $W$ is updated such that the projection $y=W Q^{T} x$ maximizes the distance of the pdf (probability density function) to a Gaussian, where $Q$ is the whitening matrix. This distance is measured by the negentropy $J(\mathrm{y}))$ (Hyvärinen et al., 2001), which is expressed as follows.

$$
J(y) \propto[E\{G(y)\}-E\{G(v)\}]^{2}
$$

Here, $v$ is a zero mean Gaussian variable with unit variance and $G$ is some nonquadratic function. We must run the FastICA algorithm repeatedly (i.e., up to the dimensionality of the collected data) to estimate the desired number of independent components.

\section{Jade algorithm}

The joint approximation diagonalization of eigen matrices (JADE) algorithm (Cardoso and Souloumiac, 1993) takes the cumulant to find the mixing matrix. First, the whitening matrix $Q$ and signal $z=Q x$ are estimated. Next, the cumulants of the whitened mixtures $Q_{z}$ are computed. An estimate of the unitary matrix $U$ is obtained by maximizing the criteria $\lambda_{z} V_{i}$ by means of joint diagonalization. If $\lambda_{z} V_{i}$ is not exactly jointly diagonalizable, the maximization of the criteria defines a joint approximate diagonalization. An orthogonal contrast is optimized by finding the rotation matrix $U$ such that the cumulant matrices are as diagonal as possible.

$U=\arg \min \sum_{i} O f f\left(U^{T} \hat{Q}_{z} U\right)$,

The mixing matrix $A$ is calculated as $A=U Q^{-1}$, and the independent components are estimated as $y=W x=V^{T} z$, where $W=U^{T} Q$.

\section{Infomax algorithm}

The algorithm proposed by Amari et al. (1996) takes mutual information $I(\mathrm{X} ; \mathrm{Y})$, which is minimized using the natural gradient technique, thus maximizing the independence between components. Mutual information is given by

$$
I(X, Y)=H(X)-H(X \mid Y),
$$

where $H(X \mid Y)$ is the conditional entropy and $H(\mathrm{X})$ is the entropy of $X$. The conditional entropy is given by $H(X ; Y)=H(X, Y)-H(Y)$. Kullback-Leiber $(\mathrm{KL})$ divergence can also be used to the same end. The KL divergence minimization uses an algorithm to estimate the separation matrix

$$
W_{(t+1)}=W_{t}+\eta_{(t)}\left[I-g(y) y^{T}\right] W_{(t)}
$$

where $t$ represents a given approximation step and $g($.$) is a nonlinearity selected according to the output$ signals, i.e., generally, it is used for super-Gaussian distributions $g(y)=\tanh (y)$ and for sub-Gaussian distribution cases $g(y)=y$ - $\tanh (y)$.

\section{One-class Support Vector Machines}

Support vector machines (SVM) and related kernel methods have become increasingly popular tools for data mining tasks, such as classification, regression, and novelty detection (Bennet and Campbell, 2000). Statistical classification can be achieved in multiple ways (Zhuang and Dai, 2006). Typically, multiclass classifiers that use data discriminatively to derive class boundaries are selected. Alternatively, one can independently construct a single classifier for each class using only data for the class and iterating until all classes are covered. This methodology does not use discriminative information in data; however, when there is inbalance between classes, this method may provide some advantages (Zhuang and Dai, 2006), i.e., it may improve poor results (Scholkopf et al., 2001). A common problem with discriminative approaches is that the decision boundary created by, for example, a two-class SVM, can result in a significant misclassification rate if they are not well separable (Kung, 1993). 
A one-class SVM constructs a classifier from only a set of labeled positive patterns called positive training samples (Burges, 1998; Manevitz and Yousef, 2001). The one-class SVM strategy maps data into the feature space and then uses a hypersphere to describe the data. Therefore, this method requires information from a single class, and imbalanced datasets can be used without performance issues (Tran et al., 2003).

The process begins with a training set of points and assumes that a dataset has a probability distribution $P$ in the feature space. Here, the goal is to find a subset $S$ of the feature space such that the probability of a point $P$ outside $S$ is determined by an a prior condition specified by Equation 5 .

$v \in(0,1)$,

The solution to this problem is obtained by the estimation of a function $f$, which is positive in $S$ and negative in the complement $S$. Schölkopf et al. (2001) developed an algorithm that returns a function $f$. This function takes values of +1 in a small region, i.e., the hypersphere, by capturing the largest number of data and takes values of -1 everywhere else.

$$
f(x)= \begin{cases}+1 & \text { if } x \in S \\ -1 & \text { if } x \in \bar{S}\end{cases}
$$

The algorithm can be summarized as a mapping of data into a feature space $H$ by using an appropriate kernel function. The algorithm then attempts to separate data mapped from the source to a maximum margin.

In our context, we have training samples $x 1, x 2, \ldots, x l$ that belong to class $X$, where $X$ is a small subset of $R^{N}$. Here, let $\Phi: X \rightarrow H$ be the kernel that transforms the training samples to another space. Thus, to separate the set of source data, the following objective function in the primary form is minimized: $\min r^{2}-\rho+\frac{l}{v l} \sum_{i} \zeta_{i}$

subject to

$$
\| \Phi\left(x_{i}\right)-c^{2} \leq r^{2}+\zeta_{i}, \zeta_{i} \geq 0 \text { to } i \in[l]
$$

where $\mathrm{v} \in[0,1]$ represents the total number of training samples, $r$ is an orthogonal array that separates the training samples from the source to a threshold $\rho, l$ is the part of the training data rejected by the hypersphere, and $\Phi$ is used to reject the hypersphere training samples.

This optimization problem is solved with Lagrange multipliers. (Scholkopf and Smola, 2002).

$$
\begin{aligned}
& L(r, \zeta, c, \alpha, \beta)=r^{2}+\sum_{i=1}^{l} \alpha_{i}\left[\Phi\left(x_{i}\right)-c^{2}-r^{2}-\zeta_{i}\right] \\
& +\frac{l}{v l} \sum_{i=1}^{l} \zeta_{i}-\sum_{i=1}^{l} \beta_{i} \zeta_{i}
\end{aligned}
$$

$\frac{\partial L}{\partial r}=2 r\left(1-\sum \alpha_{i}\right)=0 \Rightarrow \sum \alpha_{i}=1$,

$\frac{\partial L}{\partial \zeta_{i}}=\frac{l}{v l}-\alpha_{i}-\beta_{i}=0 \Rightarrow 0 \leq \alpha_{i} \leq \frac{l}{v l}$,

$\frac{\partial L}{\partial c}=-\sum 2 \alpha_{i}\left(\Phi\left(x_{i}\right)-c\right)=0$

$\Rightarrow c=\sum \alpha_{i} \Phi\left(x_{i}\right)$.

Equations 7 and 8 lay out the rejected hyper hold training samples, and Equation 9 reports $c$ (i.e., the center of the hypersphere), which can be expressed as the linear combination $\Phi(X)$ and can be solved in a dual form with the following optimization:

$\min \sum_{i, j} \alpha_{i} \alpha_{j} K\left(x_{i}, x_{j}\right)-\sum_{i}\left(x_{i}, x_{i}\right)$

subject to

$0 \leq \alpha_{i} \leq \frac{l}{v l}, \sum_{i} \alpha_{i}=1$.

An important family of kernels is the radial basis function (RBF) used in the proposed method. The $\mathrm{RBF}$ is very commonly used in pattern recognition problems and is defined by

$k(x, y)=e^{-\gamma x-y^{2}}$

where $\gamma>0$ is a user-defined bandwidth parameter (Scholkopf and Smola, 2002).

To evaluate classifier performance, it is necessary to quantify its sensitivity, specificity, and accuracy. In the diabetic classification problem, sensitivity measures the accuracy of the classifier to identify diabetics in the population, and specificity measures the accuracy of the classifiers to identify healthy people in the population. The validation is done using the following quantities:

1. True-positive (TP): Diagnosis of patients correctly classified as diabetic.

2. False-positive (FP): Diagnosis of nondiabetic patients classified as diabetic.

3. True-negative $(\mathrm{TN})$ : Diagnosis of patients correctly classified as nondiabetic.

4. False-negative (FN) Diagnosis of diabetic patients classified as nondiabetic.

and the accuracy of the classifier is defined by:

Sensitivity: $\quad \mathrm{TP} /(\mathrm{TP}+\mathrm{FN})$

Specificity: $\quad \mathrm{TN} /(\mathrm{TN}+\mathrm{FP})$

Accuracy: $\quad(\mathrm{TP}+\mathrm{TN}) /(\mathrm{TP}+\mathrm{TN}+\mathrm{FP}+\mathrm{FN})$ 


\section{Database descriptions}

Here, we have used three databases. The first is the PIMA database (Blake and Merz, 1996), which has the disadvantage of being composed only of Indian females, which reduces the possibility of generalizing the results. To diminish this drawback, we have used two additional databases: a Brazilian database (Brasil, 2014) and an African-American database (Wang, 2014). These databases are composed of both genders, and the Brazilian data includes multiracial population. The PIMA database was obtained from the UCI (University of California, Irvine) repository of machine learning databases (Blake and Merz, 1996). This database was selected from a larger database held by the National Institutes of Diabetes and Digestive and Kidney Diseases. All patients were females aged at least 21 years of Pima Indian heritage.

We labeled the results as ' 0 ' or ' 1 ,' where ' 1 ' is a positive test for diabetes and ' 0 ' is a negative test. There were $268(34.9 \%)$ cases in class ' 1 ' and $500(65.1 \%)$ cases in class ' 0 .' In addition, there were eight clinical indicators:

1. Number of pregnancies.

2. Plasma glucose concentration determined by a 2-hour oral glucose tolerance test.

3. Diastolic blood pressure ( $\mathrm{mmHg}$ ).

4. Tricep skin fold thickness $(\mathrm{mm})$.

5. 2-hour serum insulin ( $\mathrm{mu} \mathrm{U} / \mathrm{ml})$.

6. Body mass index.

7. Diabetes pedigree function.

8. Age (years).

Data of the Brazilian database (Brazilian Unique Health System) (Brasil, 2014) comprised patients between 12 and 100 years. There were $500(50 \%)$ cases classified as diabetics and $500(50 \%)$ as nondiabetics. There were 14 noninvasive clinical indicators:

1. Age (years).

2. Systolic blood pressure.

3. Diastolic blood pressure.

4. Hip (cm).

5. Weight $(\mathrm{Kg})$.

6. Height $(\mathrm{cm})$.

7. Family antecedent of diabetes.

8. Tobacco use.

9. Sedentary lifestyle.

10. Overweight.

11. Heart attack.

12. Other coronapathies.

13. Stroke.

14. Amputation.

Clinical indicators 1 to 6 are continuous variables, and indicators 7 to 14 are discrete variables.

The African-American database was obtained from the University of Virginia's School of Medicine. Data consists of 19 variables on 403 subjects obtained from a study conducted to understand the prevalence of obesity, diabetes, and other cardiovascular risk factors in central Virginia for African-Americans (Wang, 2014). There were $60(14.88 \%)$ cases classified as diabetics and $343(85.11 \%)$ as nondiabetics. Seven noninvasive clinical indicators were used in this database:

1. Age.

2. Weight (pounds).

3. Height (inches).

4. First systolic blood pressure $(\mathrm{mmHg})$.

5. First diastolic blood pressure ( $\mathrm{mmHg}$ ).

6. Waist (inches).

7. Hip (inches).

\section{Results}

We performed different experiments to test the proposed methodology. For the PIMA database, we used all eight indicators shown in Table 2 . Twenty percent of the 268 diabetic cases were used for training data, and the remaining $80 \%$ were used for test data, using the 20 -fold cross-validation method (in a randomized fashion). For the nondiabetic case, we have used all 500 cases for testing data because it is not necessary to train the one-class SVM for both classes. Clinical indicators can be divided into two groups, invasive or noninvasive. In this case, indicators $1,3,4,6,7$, and 8 are noninvasive indicators, and indicators 2 and 5 are invasive. We applied ICA decomposition on the full set using the FastICA algorithm and obtained matrix $A$ with eight basis functions. Then, we projected the samples on the basis functions. These projections were input to the one-class SVM. A library for SVM development (i.e., LIBSVM (Chang and Lin, 2003)) was used for training and testing. The Gaussian gamma value was set at 0.00781 which was determined by cross-validation (cost value of 0.03125$)$.

To illustrate the clustering, although we have eight features, we show the results after PCA processing in Figure 2a, for three features. In Figure $2 b$ we plot three diferent features taken from the ICA output.

Table 2. The PIMA database indicators used in experiments $(\mathrm{All}=$ all features, $6 \mathrm{f}=$ noninvasive features).

\begin{tabular}{lll}
\hline \multicolumn{1}{c}{ PIMA database clinical indicators } & All & 6f \\
\hline 1. No. of pregnancies & $\mathrm{X}$ & $\mathrm{X}$ \\
2. Glucose concentration & $\mathrm{X}$ & \\
3. Diastolic blood pressure & $\mathrm{X}$ & $\mathrm{X}$ \\
4. Triceps skin thickness & $\mathrm{X}$ & $\mathrm{X}$ \\
5. 2-hour serum insulin & $\mathrm{X}$ & \\
6. Body mass index & $\mathrm{X}$ & $\mathrm{X}$ \\
7. Pedigree function & $\mathrm{X}$ & $\mathrm{X}$ \\
8. Age & $\mathrm{X}$ & $\mathrm{X}$ \\
\hline
\end{tabular}


Last, we show in Figure 2c three features found by SVM. In this later case, it is important to remember that the data was preprocessed by PCA and ICA.

In the next experiment, the same process was applied to estimate the feature space by using ICA algorithms; however, we withdrew the invasive indicators (blood tests). The PIMA database indicators used in experiments are shown in Table 2. Moreover, six basis functions were selected to make the projection and create the input for the one-class SVM classifier, as shown in Tables 2 and 3. In addition, the Receiver operating characteristic (ROC) curves and results for the three ICA algorithms tests (FastICA, JADE and INFOMAX) without invasive indicators are shown in Figure 3 and Table 3, respectively.

To explore the generalization of the proposed methodology the Brazilian and African-American databases were tested. In this case, for each data base, considering only the noninvasive indicators during feature extraction phase, the FastICA algorithm was used. The results are shown in Table 4.

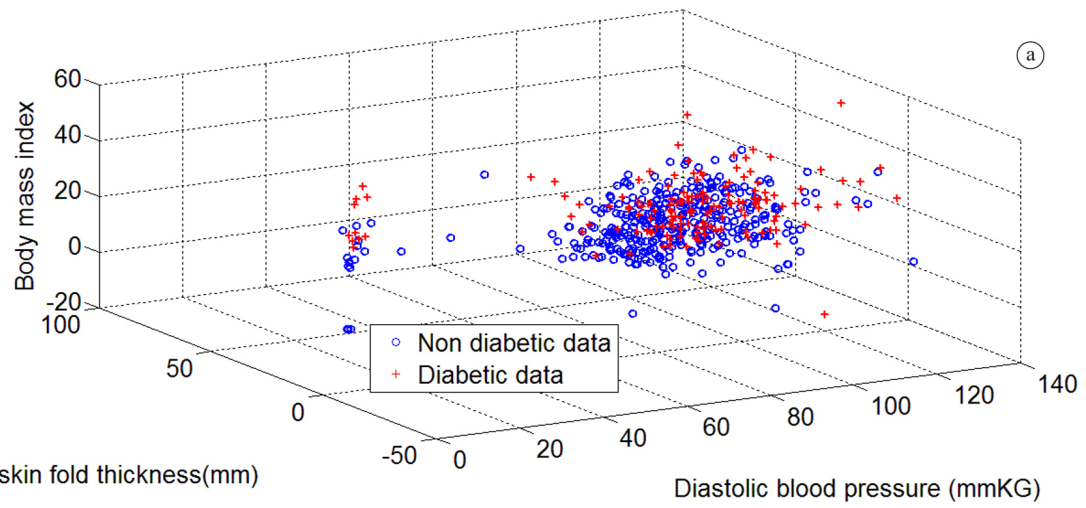

Tripec skin fold thickness $(\mathrm{mm})$

Diastolic blood pressure (mmKG)

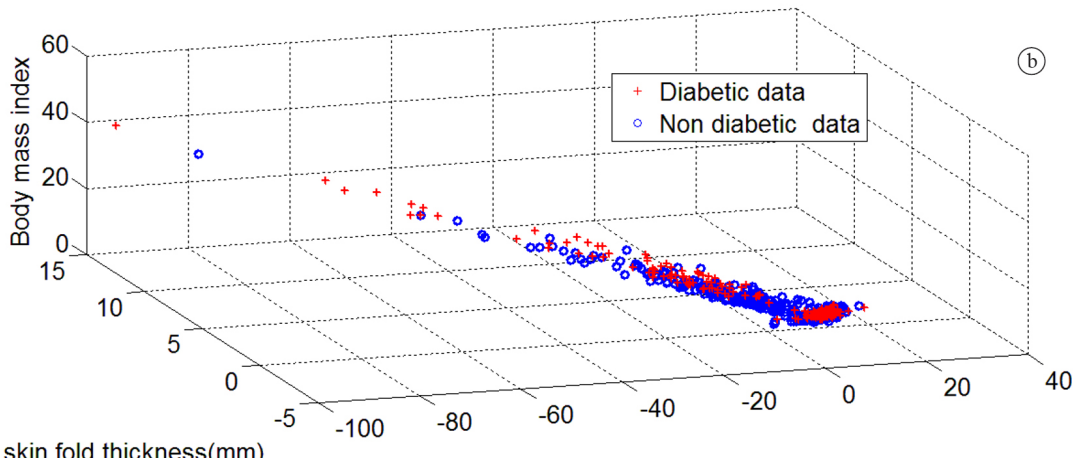

Tricep skin fold thickness $(\mathrm{mm})$

Diastolic blood pressure $(\mathrm{mmKg})$

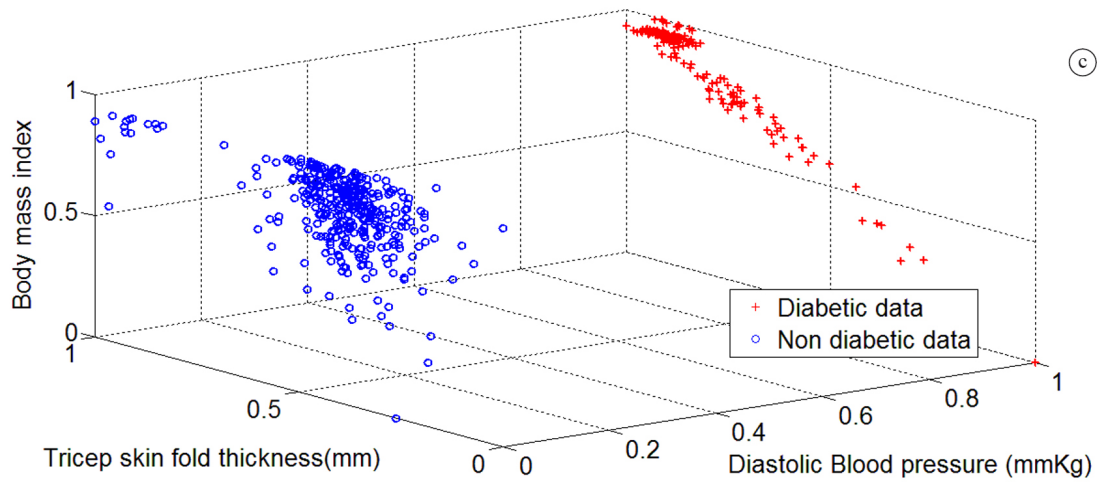

Figure 2. Three dimensional plot of three different features taken out of the eight original data.(a) three principal components found by PCA, whereas we show the diabetics in red and the nondiabetics in blue; (b) three independent components found by ICA; c) SVM (after PCA and ICA). 
Table 3. Results of ICA algorithms followed by one-class SVM using the features described in Table 2 (PIMA database). For noninvasive use (6f) an extraction was made with the three ICA algorithms most commonly used in the literature, the results are described herein.

\begin{tabular}{ccccc}
\hline & Sensitivity & Specificity & Accuracy & 95\% Confidence Level \\
\hline All (FastICA) & $99.81 \%$ & $98.34 \%$ & $98.47 \%$ & 0.22 \\
6 f (FastICA) & $99.05 \%$ & $98.19 \%$ & $98.28 \%$ & 0.39 \\
6 f (JADE) & $99.92 \%$ & $99.51 \%$ & $99.57 \%$ & 0.32 \\
6 f (InfoMAX) & $99.46 \%$ & $99.35 \%$ & $99.37 \%$ & 0.27 \\
\hline
\end{tabular}

Table 4. Test results of FastICA algorithm and one-class SVM for Brazilian data (12 features) and African-American data (five features).

\begin{tabular}{ccccc}
\hline Database & Sensitivity & Specificity & Accuracy & 95\%Confidence Level \\
\hline Brazilian & $99.99 \%$ & $99.63 \%$ & $99.81 \%$ & 0.13 \\
African-American & $98.88 \%$ & $96.68 \%$ & $97.01 \%$ & 0.36 \\
\hline
\end{tabular}

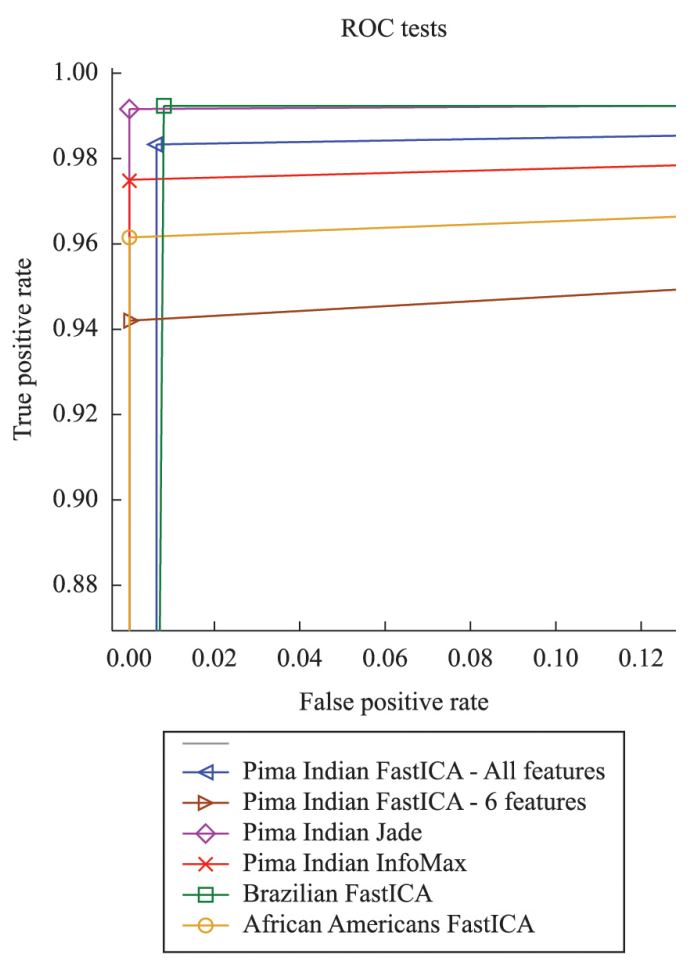

Figure 3. ROC curves for different algorithms (FastICA, InfoMax, and Jade) and different databases.

\section{Discussion}

In this paper, we presented the use of an efficient coding technique to select features to classify patients as diabetic or nondiabetic. We used a framework based on three steps consisting of applying a PCA algorithm to whiten the data, followed by an ICA algorithm, which are both based on linear operations. We then applied a one class SVM classifier as can be observed clearly in Figure 2. Figure 2a shows that PCA could not cluster data appropriately. Figure $2 \mathrm{~b}$ shows that ICA could group data more efficiently. However, we could only completely cluster data shown in Figure 2c after the SVM classifier. This can be understood in the following way. Let us firstly remember that we have two steps: the ICA training and the SVM classifier. We estimate the ICA basis functions either using the original diabetic or the non-diabetic database. It is important to emphasize that, while estimating the ICA basis functions, the original data is centered at zero (zero mean). Afterwards, we project the ICA decomposed data on the basis functions again to avoid the known scale and ordering indeterminancies. This leads to results shown in Figure 2c, where we can clearly see two well separated clusters. This occurs because each class in this data set happens to have its own mean that was found by the ICA preprocessing.

Note that other methods reported in the literature also used the same PIMA database. These methods included all data, i.e., both noninvasive and invasive clinical findings, as can be seen in first row of Table 3 and in Table 1. Our proposed method achieved an accuracy of $98.47 \%$, but the others methods achieved lower accuracy. In addition, we conducted experiments without invasive data, such as insulin and glucose concentration. In this framework, we obtained an accuracy rate of $98.28 \%$ with the FastICA algorithm, as show in second row of Table 3. This indicates that even without invasive characteristics that are decisive in diabetes diagnosis, we can maintain very high classification accuracy, although slightly lower. The same trend is observed with other ICA algorithms; we obtained an accuracy of $99.57 \%$ with the JADE algorithm and $99.37 \%$ with the InfoMAX, see the third and fourth row of Table 3, respectively. To determine the consistency of this finding, we applied the proposed technique to two other databases, i.e., Brazilian and African-American databases, as is shown in Table 4. Equivalent results to those of the PIMA database were found, as is shown in Table 3 and Table 4. Although we obtained similar accuracy when 
we removed features, Figure 3 shows that we obtained smaller values for the true positive rate when we used only six features.

One question that might arise is why does the use of efficient coding yield higher accuracy than PCA or other methods (Table 1). The efficient coding strategy finds a projection space that, in contrast to the space found by PCA, projects the data cloud in a way that it achieves as close as possible mutual statistically independent across dimensions. The characteristics of diabetics and non-diabetics happened to have different class means in the new space, which improved the cluster separation that was exploited by the classifier.

The contributions of this paper can be described as follows: 1) A method was proposed to increase performance in classifying diabetic and non-diabetics in relation to other studies in literature, as can be seen in Table 1.2) New tests using exclusively non-invasive features were conducted, and similar results were achieved when using all the features (invasive and non-invasive) (see Table 3). This is significant because it increases the possibility of tracking the disease in remote areas, at low cost and in a reliable manner, because without the invasive features (which are obtained through procedures with considerable costs for screening, for example), data can be collected with simple devices. 3) The generalization of the method in relation to diabetes was also tested on two new databases, of African-Americans and Brazilians, the latter of which had not been used in any other study of this kind in literature. The findings from these new tests are similar to those from other tests using the PIMA database (see Table 4).

Efficient coding was the key to simplify the processing complexity of the original features in a new simple and robust representation. This new space can be seen as a representation of what independently describes diabetics and non-diabetics, because the new representation is concise and non-redundant.

\section{References}

Amari S, Cichocki A, Yang HH. A new learning algorithm for blind signal separation. In: Mozer MC, Jordan MI, Petsche T, editors. Advances in Neural Information Processing Systems 9 (NIPS 1996). Cambridge: MITPress; 1996. p. 757-63.

Baddeley R, Abbott LF, Booth MC, Sengpiel F, Freeman T, Wakeman EA, Rolls ET. Responses of neurons in primary and inferior temporal visual cortices to natural scenes. Proceedings of the Royal Society of London. Series B, Biological Sciences 1997; 264(1389):1775-83. http://dx.doi. org/10.1098/rspb.1997.0246. PMid:9447735

Barros AK, Chichocki A. Neural coding by redundancy reduction and correlation. In: Proceedings of the VII Brazilian
Symposium on Neural Networks; 2002 Oct 26-30, Salvador, Bahia: SBRN-IEEE. 2002. p. 223-6.

Bennet KP, Campbell C. Support vector machines: hype or hallelujah? ACM SIGKDD Explorations Newsletter. 2000; 2(2):1-13. http://dx.doi.org/10.1145/380995.380999.

Blake CL, Merz CJ. UCI repository of machine learning databases [Internet]. 1996 [cited 2010 Aug]. Available from: http: //www.ics.uci.e.,du/ mlearn/MLRepository.html.

Brasil. Ministério da Saúde. Departamento de Informática do SUS - DATASUS. SISHiperdia [Internet]. 2014. [cited 2014 Dec]. Available from: http://hiperdia.datasus.gov.br/.

Burges CJC. A tutorial on support vector machines for pattern recognition. Data Mining and Knowledge Discovery 1998; 2(2):121-67. http://dx.doi.org/10.1023/A:1009715923555.

Byeon B, Rasheed K, Doshi P. Enhancing the quality of noisy training data using a genetic algorithm and prototype selection. In: Proceedings of the 2008 International Conference on Artificial Intelligence; 2008 July 14-17, Las Vegas, Nevada. 2008. p. 821-7.

Çalişir D, Doğantekin E. An automatic diabetes diagnosis system based on LDA wavelet support vector machine classifier. Expert Systems with Applications 2011;38(7):83115. http://dx.doi.org/10.1016/j.eswa.2011.01.017.

Cardoso JF, Souloumiac A. Blind beamforming for nongaussian signals. IEE Proceedings. Part F. Radar and Signal Processing 1993; 140(6):362-70. http://dx.doi. org/10.1049/ip-f-2.1993.0054.

Carvalho BPRD, Braga AP. IP-LSSVM: A two-step sparse classifier. Pattern Recognition Letters 2009; 30(16):1507-15. http://dx.doi.org/10.1016/j.patrec.2009.07.022.

Chang CC, Lin CJ. LIBSVM - A Library for Support Vector Machines [Internet]. 2003. [cited 2008 Jan]. Available from: http://www.csie.ntu.edu.tw/cjlin/libsvm/.

Chikh MA, Saidi M, Settouti N. Diagnosis of diabetes diseases using an Artificial Immune Recognition System2 (AIRS2) with fuzzy K-nearest neighbor. Journal of Medical Systems 2012; 36(5):2721-9. http://dx.doi.org/10.1007/ s10916-011-9748-4. PMid:21695498

Comon P. Independent component analysis, a new concept? Signal Processing 1994; 36(3):287-314. http://dx.doi. org/10.1016/0165-1684(94)90029-9.

Costa DD, Campos LF, Barros AK. Classification of breast tissue in mammograms using efficient coding. Biomedical Engineering Online 2011; 10(55):2-14. http://dx.doi. org/10.1186/1475-925X-10-55. PMid:21702953

DeWeese MR, Wehr M, Zador AM. Binary spiking in auditory cortex. The Journal of Neuroscience 2003; 23(21):7940-9. PMid:12944525.

Dogantekin E, Dogantekin A, Avci D, Avci L. An intelligent diagnosis system for diabetes on linear discriminant analysis and adaptive network based fuzzy inference system: LDAANFIS. Digital Signal Processing 2010; 20(4):1248-55. http://dx.doi.org/10.1016/j.dsp.2009.10.021.

Doi E, Inui T, Lee TW, Wachtler T, Sejnowski TJ. Spatiochromatic receptive field properties derived from 
information-theoretic analyses of cone mosaic responses to natural scenes. Neural Computation 2003; 15(2):397417. http://dx.doi.org/10.1162/089976603762552960. PMid:12590812

Ghazavi SN, Liao TW. Medical data mining by fuzzy modeling with selected features. Artificial Intelligence in Medicine 2008; 43(3):195-206. http://dx.doi.org/10.1016/j. artmed.2008.04.004. PMid:18534831

Hild KE 2nd, Erdogmus D, Torkkola K, Principe JC. Feature extraction using information-theoretic learning. IEEE Transactions on Pattern Analysis and Machine Intelligence 2006; 28(9):1385-92. http://dx.doi.org/10.1109/ TPAMI.2006.186. PMid:16929726

Hubel DH, Wiesel TN. Receptive fields, binocular interaction and functional architecture in the cat's visual cortex. The Journal of Physiology 1962; 160(1):106-54. http://dx.doi. org/10.1113/jphysiol.1962.sp006837. PMid:14449617

Hyvärinen A, Karhunen J, Oja E. Independent component analysis. John Wiley and Sons: 2001.. http://dx.doi. org/10.1002/0471221317.

Hyvärinen A, Oja E. Independent component analysis: algorithms and applications. Neural Networks 2000; 13(45):411-30. http://dx.doi.org/10.1016/S0893-6080(00)00026-5. PMid:10946390

Jeatrakul P, Wong KW, Fung CC. Data cleaning for classification using misclassification analysis. Journal of Advanced Computational Intelligence and Intelligent Informatics. 2010; 14(3):297-302.

Kahramanli H, Allahverdi N. Design of a hybrid system for the diabetes and heart diseases. Expert Systems with Applications 2008; 35(1-2):82-9. http://dx.doi.org/10.1016/j. eswa.2007.06.004.

Kayaer K, Yildirim T. Medical diagnosis on Pima Indian diabetes using general regression neural networks. In: Proceedings of Joint International Conference ICANN/ ICONIP; 2003 June 26-29; Istanbul, Turkey. Springer; 2003. p.181-4.

Kung SY. Digital neural networks. 1th ed. Englewood Cliffs: Prentice Hall; 1993.

Lee C-S, Wang M-H. A fuzzy expert system for diabetes decision support application. IEEE Transactions on Man and Cybernetics, Part B. 2011; 41(1):139-53. http://dx.doi. org/10.1109/TSMCB.2010.2048899.

Lekkas S, Mikhailov L. Evolving fuzzy medical diagnosis of Pima Indians diabetes and of dermatological diseases. Artificial Intelligence in Medicine 2010; 50(2):117-26. http:// dx.doi.org/10.1016/j.artmed.2010.05.007. PMid:20566274

Li D, Liu C. A class possibility based kernel to increase classification accuracy for small data sets using support vector machines. Expert Systems with Applications 2010; 37(4):3104-10. http://dx.doi.org/10.1016/j.eswa.2009.09.019.

Lucena F, Barros AK, Príncipe JC, Ohnishi N. Statistical coding and decoding of heartbeat intervals. PLoS ONE 2011; 6(6):e20227. http://dx.doi.org/10.1371/journal. pone.0020227. PMid:21694763
Luukka P. Feature selection using fuzzy entropy measures with similarity classifier. Expert Systems with Applications 2011a; 38(4):4600-7. http://dx.doi.org/10.1016/j.eswa.2010.09.133.

Luukka P. Fuzzy beans in classification. Expert Systems with Applications 2011b; 38(5):4798-801. http://dx.doi. org/10.1016/j.eswa.2010.09.167.

Manevitz L, Yousef M. One-class SVMs for document classification. Journal of Machine Learning Research 2001; 2(2):139-54.

Mat Isa NA, Mamat WMFW. Clustered-hybrid multilayer perceptron network for pattern recognition application. Applied Soft Computing 2011; 11(1):1457-66. http://dx.doi. org/10.1016/j.asoc.2010.04.017.

Miche Y, Sorjamaa A, Bas P, Simula O, Jutten C, Lendasse A. OP-ELM: optimally pruned extreme learning machine. IEEE Transactions on Neural Networks 2010; 21(1):158-62. http:/ dx.doi.org/10.1109/TNN.2009.2036259. PMid:20007026

Patil BM, Joshi RC, Toshniwal D. Hybrid prediction model for type-2 diabetic patients. Expert Systems with Applications 2010; 37(12):8102-8. http://dx.doi.org/10.1016/j. eswa.2010.05.078.

Polat K, Gunes S, Arslan A. A cascade learning system for classification of diabetes disease: generalized discriminant analysis and least square support vector machine. Expert Systems with Applications 2008; 34(1):482-7. http://dx.doi. org/10.1016/j.eswa.2006.09.012.

Polat K, Güneş S. An expert system approach based on principal component analysis and adaptive neuro-fuzzy inference system to diagnosis of diabetes disease. Digital Signal Processing 2007; 17(4):702-10. http://dx.doi. org/10.1016/j.dsp.2006.09.005.

Reddy MB, Reddy LSS. Dimensionality reduction: an empirical study on the usability of IFECF (independent feature elimination- by c-correlation and f- correlation) measures. International Journal of Computer Science. 2010; 7(1):74-81.

Sample PA, Boden C, Zhang Z, Pascual J, Lee TW, Zangwill LM, Weinreb RN, Crowston JG, Hoffmann EM, Medeiros FA, Sejnowski T, Goldbaum M. Unsupervised machine learning with independent component analysis to identify areas of progression in glaucomatous visual fields. Investigative Ophthalmology \& Visual Science 2005; 46(10):3684-92. http://dx.doi.org/10.1167/iovs.04-1168. PMid:16186350

Schölkopf B, Platt JC, Shawe-Taylor J, Smola AJ, Williamson RC. Estimating the support of a high-dimensional distribution. Neural Computation 2001; 13(7):1443-71. http://dx.doi. org/10.1162/089976601750264965. PMid:11440593

Scholkopf B, Smola AJ. Learning with kernels: support vector machines, regularization, optimization, and beyond. Cambridge: MITPress; 2002.

Silva RN, Ferreira ACBH, Ferreira DD, Barbosa BHG. Noninvasive method to analyse the risk of developing diabetic foot. Healthcare Technology Letters. 2014; 1(4):109-13. http://dx.doi.org/10.1049/htl.2014.0076.

Simoncelli EP, Olshausen BA. Natural image statistics and neural representation. Annual Review of Neuroscience 
2001; 24(1):1193-216. http://dx.doi.org/10.1146/annurev. neuro.24.1.1193. PMid:11520932

Smith EC, Lewicki MS. Efficient auditory coding. Nature 2006; 439(7079):978-82. http://dx.doi.org/10.1038 nature04485. PMid:16495999

Tran Q, Zhang Q, Li X. Evolving training model method for one-class SVM systems. In: SMC '03 Conference Proceedings - Proceedings of the 2003 IEEE Internation
Conference on Systems, Man and Cybernetics; 2003 Oct 5-8, 2003; Washington. 2003 p. 2388-93.

Wang L. Datasets [Internet]. Vanderbilt University; 2014 [cited July 2014]. Available from: http://biostat.mc.vanderbilt. edu/wiki/Main/DataSets?CGISESSID=10713f6d891653d dcbb7ddbdd9cffb79.

Zhuang L, Dai H. Parameter optimization of kernel-based oneclass classifier on imbalance learning. Journal of Computers 2006; 1(7):32-40. http://dx.doi.org/10.4304/jcp.1.7.32-40.

\footnotetext{
Authors

Áurea Celeste Ribeiro ${ }^{1,2 *}$, Allan Kardec Barros ${ }^{2}$, Ewaldo Santana ${ }^{1,2}$, José Carlos Príncipe ${ }^{3}$

${ }^{1}$ Programa de Pós-Graduação em Engenharia de Computação e Sistemas, Universidade Estadual do Maranhão - UEMA,

Cidade Universitária Paulo VI, Caixa Postal 09, CEP 65055-310, São Luís, MA, Brazil

${ }^{2}$ Laboratório de Processamento da Informação Biológica, Universidade Federal do Maranhão - UFMA,

Campus Bacanga, Av. dos Portugueses, s/n, CEP 65080-805, São Luís, MA, Brazil

${ }^{3}$ Computational NeuroEngineering Laboratory, University of Florida, EB451 Engineering Building,

PO Box 116130, Gainesville, FL, USA, 32611
} 